\title{
Feebly Generalized Closed Sets in Bitopological Spaces
}

\author{
K.Bala Deepa Arasi, S.V.Vani, V.Maheswari
}

Abstract: In this paper, Feebly generalized closed sets (fg-closed sets) in bitopological spaces is introduced and the properties are analysed. As an application, the notion of $\tau_{1} \tau_{2}-T_{f g}$ space, $\tau_{1} \tau_{2} *{ }_{T_{f g}}$ space, $\tau_{1} \tau_{2}-g \alpha g T_{f g}$ space are investigated.

Keywords: $\tau_{1} \tau_{2}-f g$ closed sets, $\tau_{1} \tau_{2}-T_{f g}, \tau_{1} \tau_{2}-*_{T_{f g}}$, $\tau_{1} \tau_{2}-g \alpha g T_{f g}$ spaces.

2010 AMS Subject classification code: $54 E 55$

Keywords: Feebly generalized.

\section{INTRODUCTION}

A triple $\left(X, \tau_{1}, \tau_{2}\right)$ consisting of a non-empty set together with a pair of Topologies $\tau_{1} \& \tau_{2}$ on $X$, is defined to be a bitopological space by J.C.Kelly[9] as introduced "Bitopological spaces" and published in proceeding of London Mathematical Society, 1963. In 1986, T.Fukutake[6] projected the concept of $g$-closed sets for bitopological spaces through the mathematical paper "On Generalized closed sets In Bitopological spaces". In 2014, K.ChandrasekharaRao and K.Kannan[8], QaysHatem Imran[6] introduced the concept of semi star generalized closed sets, Genneralized Alpha-closed sets in bitopological spaces respectively. In 2016 K.BalaDeepaArasi, et.al[4] introduced $s b \hat{g}$-closed sets in bitopological spaces. In 2017 N.Gayathri and P.Keerthika[7] introduced $\tau_{1} \tau_{2}-g^{*} s^{*}$ closed sets. Recently Maheshwari S.N. and Jain.P.C.[12] introduced and studied feebly open sets in topological spaces. In this paper is to introduced a new class of closed sets called $\tau_{1} \tau_{2}$-Feebly generalized closed sets in Bitopological spaces.

\section{PRELIMINARIES}

Throughout this paper $X$ and $Y$ always represent non empty bitopological spaces $\left(X_{,} \tau_{1}, \tau_{2}\right)$ and $\left(Y_{,} \sigma_{1}, \sigma_{2}\right)$ on which no separation axioms are assumed unless explicitly mentioned and the integers $m, n \in\{1,2\}$. For a subset $B$ of $X$ with a topology $\tau$, the closure of $B$ is

Revised Manuscript Received on November 05, 2019.

K.Bala Deepa Arasi, Assistant Professor of Mathematics, A.P.C.Mahalaxmi College for Women, Thoothukudi, TN, India.

E.Mail : baladeepa@apcmcollege.ac.in

S.V.VANI, Research Scholar, PG\&Research Department of Mathematics, A.P.C.Mahalaxmi College for Women, Thoothukudi, TN, India. E.Mail : vanikathir16@gmail.com.

V.Maheswari, Assistant Professor of Mathematics, A.P.C.Mahalaxmi College for Women, Thoothukudi, TN, India

E.Mail : maheswari@apcmcollege.ac.in denoted by $\tau-c l(B)$ or $\operatorname{cl}(B)$, the interior of B is denoted by $\tau$-int $(B)$ or int $(B)$ and the complement of $B$ in $X$ is denoted by $B^{c}$.

\section{3. $(m, n)$ - FEEBLY GENERALIZED CLOSED} SETS

In this section, a new class of closed sets in bitopological spaces is introduced.

Definition 3.1: A subset B of a bitopological space $\left(X, \tau_{1}, \tau_{2}\right)$ is called $(m, n)-$ Feebly generalized closed if $\tau_{n}-f c l(B) \subseteq V$ whenever $\mathrm{B} \subseteq V$ and $V$ is feebly open in $\tau_{m}$.

Proposition 3.2: Every $\boldsymbol{\tau}_{n}$-closed set in $\left(X, \tau_{1}, \tau_{2}\right)$ is $(m, n)$-FG closed set but not conversely.

Proof: Assume that B is $\tau_{n}$-closed set and $V$ be any feebly open in $\tau_{m}$ containing B*

Then $\quad \tau_{n} \quad-\quad c l(B)=B \subseteq V \quad$.Therefore $\tau_{n}-f c l(B) \subseteq \tau_{n}-\operatorname{cl}(B) \subseteq V$.

Hence $B$ is $(m, n)$ - Feebly generalized closed.

Observation:

Every $(m, n)-$ Feebly generalized closed set need not be a $\tau_{n}$-closed set.

Example 3.3: Let $X=\{\alpha, \beta, \gamma\}$ be a bitopological space with topologies

$\tau_{1}=\{X, \emptyset,\{\gamma\},\{\alpha, \gamma\}\}$ and $\tau_{2}=\{X, \emptyset,\{\alpha\}\}$

Here the set $B=\{\alpha, \beta\}$ is $(1,2)-f g$ closed set but not $\tau_{2}$-closed.

Proposition 3.4: Every $(m, n)-g$ closed set is $(m, n)$ - Feebly generalized closed set. $^{2}$

Proof: Let B be any $(m, n)-g$ closed set and V be any $\tau_{m}$ - open set containing B.

Since every $\tau_{m}$-open set is $\tau_{m}-$ feebly open set. Hence $\tau_{n}-f c l(B) \subseteq \tau_{n}-c l(B) \subseteq V$.

Therefore B is $(m, n)-$ Feebly generalized closed set.

Observation: Every $(m, n)$ - Feebly generalized closed set need not be a $(m, n)-g$ closed set.

Example 3.5: Let $X=\{\alpha, \beta, \gamma\}$ be a bitopological space with topologies $\quad \tau_{1} \quad=\quad\{X, \emptyset,\{\alpha\},\{\alpha, \beta\}\}$ and $\tau_{2}=\{X, \emptyset,\{\alpha\},\{\beta\},\{\alpha, \beta\}\}$. Here the set $\mathrm{B}=\{\alpha\}$ is 
$(1,2)-f g$ closed set but not $(1,2)-g$ closed set.

Proposition 3.6: Every $(m, n)-g^{*}$ closed set is $(m, n)-$ Feebly generalized closed set.

Proof: Let $B$ be $(m, n)-g^{*}$ closed set. Let $B \subseteq V$ and $V$ is $\tau_{m}-$ feebly open. Then $V$ is $\tau_{m}-g$ open and hence $\tau_{n}-c l(B) \subseteq V$, since $\mathrm{B}$ is $(m, n)-g^{*}$ closed. Now, $\tau_{n}-f c l(B) \subseteq \tau_{n}-\operatorname{cl}(B) \subseteq V$. Hence $B$ is $(m, n)-$ Feebly generalized closed set.

\section{Observation:}

Every $(m, n)-$ Feebly generalized closed set need not be a $(m, n)-g^{*}$ closed set.

Example 3.7: Let $X=\{\alpha, \beta, \gamma\}$ be a bitopological space with topologies $\tau_{1}=\{X, \emptyset,\{\beta\}\}$ and $\tau_{2}=\{X, \emptyset,\{\alpha\},\{\alpha, \beta\}\}$. Here the set $B=\{\alpha\}$ is $(1,2)-f g$ closed set but not $(1,2)-g^{*}$ closed set.

\section{Preposition 3.8:}

$(m, n)-$ Feebly generalized closed set is $(m, n)-s^{*} g$ closed.

Proof: Let

$B$

$(m, n)-$ Feebly generalized

closed. $\tau_{n}-f c l(B) \subseteq V$ whenever $B \subseteq V$ and $V$ is feebly open in $\tau_{m}$. Also $\tau_{n}-f c l(B) \subseteq \tau_{n}-c l(B) \subseteq V$ and $V$ is $\tau_{m}-$ semi open

Hence, $B$ is $(m, n)-s^{*} \mathrm{~g}$ closed.

Observation: Every $(m, n)-s^{*} g$ closed set is need not be a $(m, n)-$ feebly generalized closed set.

Example 3.9: Let $X=\{\alpha, \beta, \gamma, \delta\}$ be a bitopological space with topologies $\tau_{1}=\{X, \emptyset,\{\alpha\}\}$ and $\tau_{2}=\{X, \emptyset,\{\alpha, \beta\},\{\alpha, \beta, \gamma\}\}$. Here the set $B=\{\alpha, \beta, \gamma\}$ is $(1,2)-s^{*} g$ closed set but not $(1,2)-f g$ closed set. Proposition 3.10: Every $(m, n)-$ Feebly generalized closed set is $(m, n)-g^{*} s$ closed.

Proof: Let $B$ be $(m, n)-$ Feebly generalized closed. Then $\tau_{n}-f c l(B) \subseteq V$ whenever $B \subseteq V$ and $V$ is feebly open in $\tau_{m}$. Now, $\tau_{n}-f c l(B) \subseteq \tau_{n}-\operatorname{scl}(B) \subseteq V$ and $V$ is

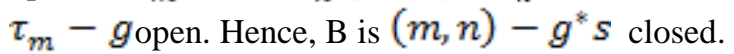

Observation: Every $(m, n)-g^{*} s$ closed set need not be a $(m, n)$ - feebly generalized closed set.

Example 3.11: Let $X=\{\alpha, \beta, \gamma\}$ be a bitopological space with topologies $\tau_{1}=\{X, \emptyset,\{\beta\},\{\beta, \gamma\}\}$ and $\tau_{2}=\{X, \emptyset,\{\alpha, \beta\}\}$. Here the set $\mathrm{B}=\{\alpha\}$ is $(1,2)-g^{*} s$ closed set but not $(1,2)-f g$ closed set.

\section{Proposition}

3.12:

Every $(m, n)-$ Feebly generalized closed set is $(m, n)-g^{*} s^{*}$ closed.

Proof: Let $B$ be $(m, n)-$ Feebly generalized closed. Then $\tau_{n}-f c l(B) \subseteq V$ whenever $B \subseteq V$ and $V$ is feebly open in $\tau_{m}$. Now, $\tau_{n}-f c l(B) \subseteq \tau_{n}-\operatorname{scl}(B) \subseteq V$ and $\mathrm{V}$ is $\tau_{m}-g^{*}$ open. Hence, $B$ is $(m, n)-g^{*} s^{*}$ closed.
Observation: Every $(m, n)-g^{*} s^{*}$ closed set is need not be a $(m, n)-$ feebly generalize closed set.

Example 3.13: Let $X=\{\alpha, \beta, \gamma\}$ be a bitopological space with topologies $\tau_{1}=\{X, \emptyset,\{\beta\}\}$ and $\tau_{2}=\{X, \emptyset,\{\alpha\},\{\beta, \gamma\}\}$.

Here the set $S=\{a, b\}$ is $(1,2)-g^{*} s$ closed set but not $(1,2)-f g$ closed set.

Proposition 3.14: Every $(m, n)-g \alpha g$ closed set is $(m, n)-$ feebly generalized closed.

Proof: Let $B$ be $(m, n)-g a g$ closed. Then $\tau_{n}-c l(B) \subseteq V$ whenever $B \subseteq V$ and $V$ is open in $\tau_{m}$. Now, $\quad \tau_{n}-f c l(B) \subseteq \tau_{n}-\operatorname{cl}(B) \subseteq V$ and $V$ is $\tau_{m}-$ feebly open. Hence, B is $(m, n)-$ feebly generalized closed.

Observation: Every $(m, n)-$ feebly generalized closed set is need not be a $(m, n)-g \alpha g$ closed set.

Example 3.15: Let $\mathrm{X}=\{\alpha, \beta, \gamma\}$ be a bitopological space with topologies $\tau_{1}=\{X, \emptyset,\{\beta\},\{\alpha, \gamma\}\}$ and $\tau_{2}=\{X, \emptyset,\{\alpha\},\{\beta\},\{\alpha, \beta\}\}$. Here the set $S=\{\alpha, \beta\}$ is $(1,2)-$ feebly generalized closed set but not $(1,2)-g \alpha g$ closed set.

Proposition 3.16: Every $(m, n)-$ feebly generalized closed set is $(m, n)-g s p$ closed.

Proof: Let $B$ be $(m, n)-$ feebly generalized closed. Then $\tau_{n}-f c l(B) \subseteq V$ whenever $B \subseteq V$ and $V$ is feebly open in $\tau_{m}$. We have $\tau_{n}-f c l(B) \subseteq \tau_{n}-\operatorname{spcl}(B) \subseteq V$ and $\mathrm{V}$ is $\tau_{m}-$ open. Therefore, B is $(m, n)-g s p$ closed.

Observation: Every $(m, n)-g s p$ closed set is need not be a $(m, n)$ - feebly generalized closed set.

Example 3.17: Let $X=\{\alpha, \beta, \gamma\}$ be a bitopological space with topologies $\tau_{1}=\{X, \emptyset,\{\beta\},\{\alpha, \beta\}\}$ and $\tau_{2}=\{X, \emptyset,\{\alpha, \gamma\}\}$. Here the set $S=\{\beta, \gamma\}$ is $(1,2)-g s p$ closed set but not $(1,2)-$ feebly generalized closed set. Proposition 3.18: Every $(m, n)-s b \hat{g}$ closed set is $(m, n)-$ feebly generalized closed.

Proof: Let B be $(m, n)-s b \hat{g}$ closed. Then $\tau_{n}-\operatorname{scl}(B) \subseteq V$ whenever $B \subseteq V$ and $V$ is open in $\tau_{m}-b \hat{g}$. We have $\tau_{n}-f c l(B) \subseteq \tau_{n}-\operatorname{scl}(B) \subseteq V$ and $\mathrm{V}$ is $\tau_{m}-$ feebly open. Therefore, $B$ is $(m, n)-$ feebly generalized closed.

Observation: Every $(m, n)-$ feebly generalized closed set is need not be a $(m, n)-s b \hat{g}$ closed set.

Example 3.19: Let $X=\{\alpha, \beta, \gamma\}$ be a bitopological space with topologies $\tau_{1}=\{X, \emptyset,\{\alpha\},\{\beta\},\{\alpha, \beta\}\}$ and $\tau_{2}=\{X, \emptyset,\{\gamma\},\{\alpha, \beta\}\}$. Here the set $S=\{\alpha, \beta\}$ is $(1,2)-$ feebly generalized closed set but not $(1,2)-s b \hat{g}_{\text {closed set. }}$

Remark 3.20: Every $(m, n)-\quad$ strongly $\alpha^{* *}$

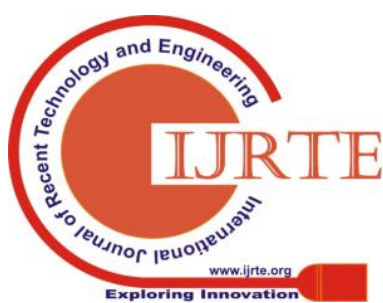


closedness is independent of $(m, n)$ - feebly generalized closedness

Example 3.21: Let $X=\{\alpha, \beta, \gamma\}$ be a bitopological space with topologies $\tau_{1}=\{X, \emptyset,\{\alpha\},\{\beta\},\{\alpha, \beta\}\}$ and $\tau_{2}=\{X, \emptyset,\{\alpha\},\{\alpha, \beta\}\}$. Here the set $S=\{\alpha, \gamma\}$ is $(1,2)-$ strongly $\alpha^{* *}$ closed set but not $(1,2)$ - feebly generalized closed. Also the set $S=\{\alpha, \beta\}$ is $(1,2)-$ feebly generalized closed but not $(1,2)-$ strongly $\alpha^{8 *}$ closed.

Remark 3.22: $(m, n)-g s$ closedness is independent of $(m, n)$ - feebly generalized closedness

Example 3.23: Let $\mathrm{X}=\{\alpha, \beta, \gamma\}$ be a bitopological space with topologies $\tau_{1}=\{X, \emptyset,\{\alpha\},\{\alpha, \beta\}\}$ and $\tau_{2}=\{X, \emptyset,\{\alpha\},\{\beta\},\{\alpha, \beta\}\}$. Here the set $S=\{\beta\}$ is $(1,2)-g s$ closed set but not $(1,2)$ - feebly generalized closed. Also the set $S=\{\alpha, \beta\}$ is $(1,2)-$ feebly generalized closed but not $(1,2)-g s$ closed.

Remark 3.24: $(m, n)-s g^{* *}$ closedness is independent of $(m, n)$ - feebly generalized closedness

Example 3.25: Let $X=\{\alpha, \beta, \gamma\}$ be a bitopological space with topologies $\tau_{1}=\{X, \emptyset,\{\alpha\},\{\alpha, \beta\}\}$ and $\tau_{2}=\{X, \emptyset,\{\alpha\},\{\beta\},\{\alpha, \beta\}\}$. Here the set $S=\{\beta\}$ is $(1,2)-s g^{* 8} \quad$ closed set but not $(1,2)-$ feebly generalized closed. Also the set $S=\{\alpha, \beta\}$ is $(1,2)-$ feebly generalized closed but not $(1,2)-s g^{* *}$ closed.

Remark 3.26: $(m, n)-g^{\# \#}$ closedness is independent of $(m, n)$ - feebly generalized closedness

Example 3.27: Let $X=\{\alpha, \beta, \gamma\}$ be a bitopological space with topologies $\tau_{1}=\{X, \emptyset,\{\alpha\},\{\alpha, \beta\}\}$ and $\tau_{2}=\{X, \emptyset,\{\alpha\},\{\beta, \gamma\}\}$. Here the set $S=\{\gamma\}$ is $(1,2)-g^{\# \#} \quad$ closed set but not $(1,2)-$ feebly generalized closed. Also the set $S=\{\alpha, \beta\}$ is $(1,2)-$ feebly generalized closed but not $(1,2)-g$ closed.

\section{BASIC PROPERTIES OF $\left(m_{n} n\right)$ - FEEBLY GENERALIZED CLOSED SETS}

Proposition 4.1: Union of any two $(m, n)-$ feebly generalized closed sets is again $(m, n)$ - feebly generalized closed set.

Proof: Let $B$ and $C$ be $(m, n)$-feebly generalized closed sets. Then $B \subseteq V$ and $C \subseteq V$ where $V$ is $\tau_{m}$-feebly open. This implies $\tau_{n}-f c l(B) \subseteq V$ or $\tau_{n}-f c l(C) \subseteq V$. Now $\tau_{n}-f c l(B \cup C) \subseteq B$, whenever $B \cup C \subseteq V$ and $V$ is feebly open in $\tau_{m}$. Therefore $B \cup C$ is $(m, n)$ - feebly generalized closed set.
Proposition 4.2: Intersection of any two $(m, n)-$ feebly generalized closed sets is again $(m, n)$ - feebly generalized closed set.

Proof: Let B and $C$ be $(m, n)$ - feebly generalized closed sets. Then $B \subseteq V$ and $C \subseteq V$ where $V$ is $\tau_{m}$-feebly open. This implies $\tau_{n}-f c l(B) \subseteq \mathrm{V}$ and $\tau_{n}-f c l(B) \subseteq V$. Now, $\quad \tau_{n}-f c l(B \cap C) \subseteq V$, whenever $B \cap C \subseteq V$ and $V$ is feebly open in $\tau_{m}$. Therefore $B \cap C$ is $(m, n)$ - feebly generalized closed set.

Remark 4.3: $(m, n)$ - feebly generalized closed set is generally not equal to $(n, m)$ - feebly generalized closed as seen from the following example.

Example 4.4: Let $X=\{\alpha, \beta, \gamma\}$ be a bitopological space with topologies $\tau_{1}=\{X, \emptyset,\{\alpha\},\{\alpha, \beta\}\}$ and $\tau_{2}=\{X, \emptyset,\{\alpha\},\{\beta\},\{\alpha, \beta\}\}$. Here, $(2,1)-f g$ closed set is $\{X, \emptyset,\{\beta\},\{\gamma\},\{\alpha, \beta\},\{\beta, \gamma\}\}$ and $(1,2)-f g$ closed set is $\{X, \emptyset,\{\alpha\},\{\gamma\},\{\alpha, \beta\},\{\alpha, \gamma\},\{\beta, \gamma\}\}$. Hence $(1,2)-f g$ closed set $\neq(2,1)-f g$ closed set.

Remark 4.5: The following diagram shows the relations among the different types of closed sets that were studied in this section.

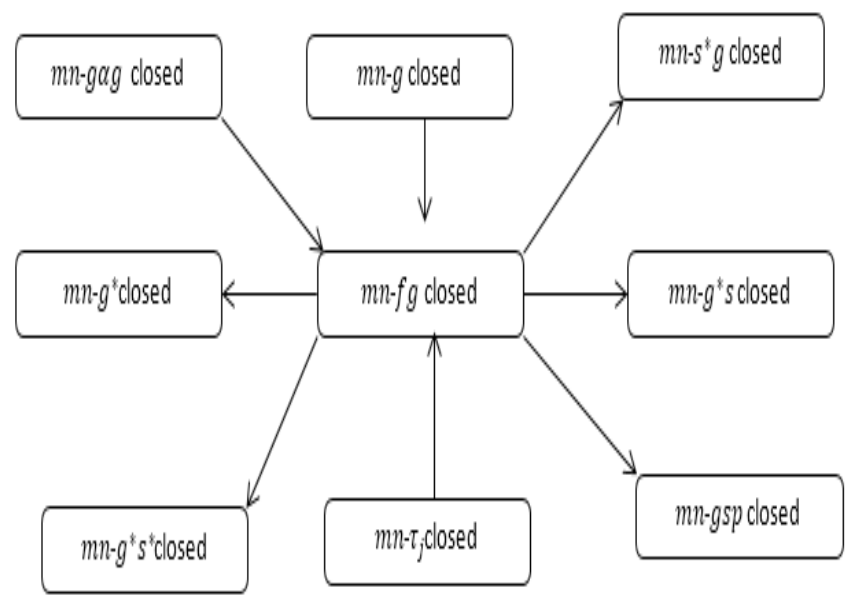

\section{CONCLUSION}

Through the above findings, this paper has attempted to compare $(m, n)-$ FG closed with the other closed sets in bitopological spaces. An attempt of this paper is to start the several definitions and results that shown in this paper, will result in obtaining several characterizations and enable to study various properties as well. It brings to limelight that the weaker form of continuity in bitopological settings is the future scope of the study. 


\section{REFERENCES}

1. K.Bala Deepa Arasi,S.Navaneetha Krishnan \&S.PiousMissier, $\left(\tau_{i}, \tau_{j}\right)-s b \hat{g}$ closed sets in Bitopological spaces, IJSAR, 3(8),2016:104-116.

2. N.Gayathri and P.Keerthika, $\tau_{1} \tau_{2}-g^{8} S^{8}$ closed sets in Bitopological spaces, IJSEC, Research Article, Volume7(2017) 10442-10446.

3. K.Kannan, D.Narasimhan and K.ChandrasekharaRao,On semi star generalized closed sets in Bitopological spaces,Bol.Soc.Paran.Mat.v.28(2010) 29-40.

4. J.C.Kelly, Bitopological spaces, Proc.London Math.Soc 13(3) (1963) 71-89.

5. Maheshwari S.N.and Jain P.C., Some New mappings, Mathematica 24(47)(1-2)(1982) 53-55.

6. Qays HatemImran, Generalized Alpha Generalized closed sets in Bitopological spaces,IRJPA,4(3),(2014) 419-425. 